\section{Inhibition of the NMC-A $\beta$-Lactamase by a Penicillanic Acid Derivative and the Structural Bases for the Increase in Substrate Profile of This Antibiotic Resistance Enzyme}

Lionel Mourey ${ }^{\dagger}{ }^{\prime}$ Kazuyuki Miyashita ${ }^{\ddagger}$ Peter Swarén, Alexey Bulychev, $₫$ Jean-Pierre Samama, ${ }^{* \dagger}$, and Shahriar Mobashery*

Groupe de Cristallographie Biologique Institut de Pharmacologie et de Biologie Structurale du CNRS 205 route de Narbonne, 31077-Toulouse Cedex, France Department of Chemistry, Wayne State University Detroit, Michigan 48202-3489

Received May 26, 1998

Bacteria resistant to multiple antibiotics have now given rise to cases which are best described as the "totally resistant" bacteria. ${ }^{1-8}$ Vancomycin (a glycopeptide) and imipenem (a carbapenem $\beta$-lactam) are considered antibiotics of last resort in the treatment of serious infections. Vancomycin is not useful against Gram-negative bacteria since it cannot gain entry into these organisms because of the barrier of the outer membrane. Multiple-resistant Gram-negative bacteria and vancomycinresistant enterococci (Gram-positive) can only be treated with imipenem

The most common mechanism of resistance to $\beta$-lactam antibiotics is the ability of bacteria to produce $\beta$-lactamases, enzymes which hydrolyze the $\beta$-lactam moiety of these antibiotics. Whereas over $250 \beta$-lactamases have been identified to date, ${ }^{9}$ imipenem has been relatively immune to the action of the most prevalent of these enzymes, those of classes A and C (both are active site serine enzymes). Regrettably, imipenem is being compromised in resistant organisms by the evolutionary advent of recently discovered $\beta$-lactamases. ${ }^{10}$ These enzymes are class $\mathrm{B} \beta$-lactamases, which are zinc-dependent, and they are also novel class A enzymes. Although the spread of the class B $\beta$-lactamases has been much feared, ${ }^{10}$ it has not become a serious threat to date. However, the novel carbapenem-hydrolyzing class A enzymes are considered a serious threat to antibacterial therapy presently. To compound the problem, these enzymes are not often inhibited by the known inhibitors of class A $\beta$-lactamases. We describe herein the first example of an inhibitor for a class A carbapenem-hydrolyzing $\beta$-lactamase, the NMC-A ("nonmetallocarbapenamase of class A"). ${ }^{11}$ Furthermore, we describe the highresolution crystal structure for the inhibitor bound to the enzyme and provide insight into the structural reason for the increased breadth of activity of this clinically important enzyme.

Table 1 summarizes the kinetic parameters for the turnover of five $\beta$-lactams for the NMC-A and TEM- $1 \beta$-lactamases; the latter

${ }^{\dagger}$ Institut de Pharmacologie et de Biologie Structurale du CNRS.

\# Wayne State University.

(1) Neu, H. C. Science 1992, 257, 1064.

(2) Levy, S. B. In Antibiotic resistance: Origins, selection and spread; Chadwick, D. J., Goode, J., Eds.; Ciba Foundation Symposium 207; Wiley: Chichester, 1997; pp 1-14.

(3) Berkowitz, F. E. South. Med. J. 1995, 88, 797.

(4) Jones, R. N. Am. J. Med. 1996, 100 (suppl. 6A), 3S

(5) Howe, R. A.; Brown, N. M.; Spencer, R. C. J. Clin. Pathol. 199649 444.

(6) Swartz, M. N. Proc. Natl. Acad. Sci. U.S.A. 1994, 91, 2420.

(7) Pinner, R. W.; Teutsch, S. M.; Simonsen, L.; Klug, L. A.; Graber, J.

M.; Clarke M. J.; Berkelman, R. L. J. Am. Med. Assoc. 1996, 275, 189.

(8) The World Health Report, World Health Organization, Geneva, 1996

(9) Bush, K.; Jacoby, G. J. Antimicrob. Chemother. 1997, 39, 1. Also, $\beta$-lactamases are listed at the following Web site: http://www.lahey.org/studies/ webt.htm.

(10) Rasmussen, B. A.; Bush, K. Antimicrob. Agents Chemother. 1997, 41,223

(11) Nordmann, P.; Ronco, E.; Naas, T.; Duport, C.; Michel-Briand, Y.; Labia, R. Antimicrob. Agents Chemother. 1993, 37, 962.
Table 1. Kinetic Parameters for Turnover of Substrates by the NMC-A and TEM-1 $\beta$-Lactamases

\begin{tabular}{llrcc}
\hline enzyme & \multicolumn{1}{c}{ compd } & $K_{\mathrm{m}}(\mu \mathrm{M})$ & $k_{\mathrm{cat}}\left(\mathrm{s}^{-1}\right)$ & $k_{\mathrm{cat}} / K_{\mathrm{m}}\left(\mathrm{M}^{-1} \mathrm{~s}^{-1}\right)$ \\
\hline NMC-A & ampicillin & $510 \pm 90$ & $140 \pm 15$ & $(2.8 \pm 0.6) \times 10^{5}$ \\
& imipinem & $340 \pm 60$ & $130 \pm 10$ & $(3.8 \pm 0.7) \times 10^{5}$ \\
& $\mathbf{1}$ & $70 \pm 50$ & $13 \pm 4$ & $(2.0 \pm 1.5) \times 10^{5}$ \\
& $\mathbf{2}$ & $600 \pm 60$ & $0.23 \pm 0.01$ & $380 \pm 40$ \\
& $\mathbf{3}$ & $a$ & $a$ & \\
TEM-1 & ampicillin $^{b}$ & $20 \pm 2$ & $1500 \pm 150$ & $(7.7 \pm 0.8) \times 10^{7}$ \\
& imipinem $^{c}$ & $27 \pm 3$ & $0.040 \pm 0.004$ & $(1.5 \pm 0.2) \times 10^{3}$ \\
& $\mathbf{1}^{d}$ & $24 \pm 3$ & $0.029 \pm 0.003$ & $(1.3 \pm 0.7) \times 10^{3}$ \\
& $\mathbf{2}$ & $e$ & $e$ & $57 \pm 5$ \\
& $\mathbf{3}$ & $e$ & $e$ & $66 \pm 2$
\end{tabular}

${ }^{a}$ Compound behaved as an inactivator for the enzyme with the following kinetic parameters: $k_{\text {inact }}=0.08 \pm 0.01 \mathrm{~s}^{-1}$ and $K_{\mathrm{I}}=390 \pm$ $70 \mu \mathrm{M} .{ }^{b}$ From ref 16. ${ }^{c}$ Zafaralla, G.; Mobashery, S. J. Am. Chem. Soc. 1992, 114, 1505. ${ }^{d}$ From ref $13 .{ }^{e}$ Individual constant determination was impossible, and the ratio of $k_{\text {cat }} / K_{\mathrm{m}}$ was determined under conditions of $K_{\mathrm{m}}>$ [S]

is the most common representative member of the Gram-negative class A enzymes. ${ }^{12}$ It would appear that the NMC-A enzyme takes a sacrifice of approximately 2 orders of magnitude in $k_{\text {cat }} /$ $K_{\mathrm{m}}$ for the typical penicillin substrate, such as ampicillin, to increase its breadth of substrate profile to include imipenem (Scheme 1). Furthermore, this enzyme turns over imipenem as efficiently as ampicillin. On the other hand, imipenem is a poor substrate for the common TEM-1 enzyme (over 4 orders of magnitude attenuation in $k_{\mathrm{cat}} / K_{\mathrm{m}}$ compared to ampicillin), which explains its clinical utility in organisms which harbor this enzyme, and others like it.

We described recently $6 \alpha$-(hydroxymethyl)penicillanate (1) as an effective inhibitor of the TEM-1 $\beta$-lactamase, in light of the fact that its turnover by the enzyme was poor (Table 1). ${ }^{13}$ The crystal structure of the enzyme modified by $\mathbf{1}$ showed that the hydroxymethyl moiety donated a hydrogen bond to the hydrolytic water molecule in the active site at the acyl enzyme intermediate stage. ${ }^{14}$ As a result, deacylation was greatly impeded, as the travel of the hydrolytic water to the ester carbonyl was prevented for steric and electrostatic reasons. In contrast, this compound is essentially as good a substrate for the NMC-A enzyme, as is imipenem (Table 1). We synthesized compounds 2 and $\mathbf{3}$ (see Supporting Information). When the steric bulk of the $6 \alpha$ hydroxymethyl substituent was increased to that of the $1 R$ hydroxyethyl and hydroxypropyl groups in penicillanate derivatives $\mathbf{2}$ and $\mathbf{3}$, respectively, the compounds both became exceedingly poor substrates for the TEM-1 enzyme. Modeling of the additional structural decorations on the $6 \alpha$ substituents in $\mathbf{2}$ and $\mathbf{3}$ into the X-ray structure of the inhibited complex of $\mathbf{1}$ with the TEM-1 $\beta$-lactamase showed that the additional methyl group(s) would cause severe steric encumbrance at the active site, consistent with the kinetic data. However, kinetic characterization of 2 and $\mathbf{3}$ with the NMC-A enzyme indicated that progressive enlargement of the $6 \alpha$ substituents resulted in poorer turnover of these compounds, such that $\mathbf{3}$ can only be thought of as an irreversible inhibitor for this enzyme (Table 1). This compound acylated the active site serine and simply did not undergo deacylation.

We determined the X-ray structure of the NMC-A $\beta$-lactamase complexed to compound $\mathbf{3}$ to study the differences seen in the

(12) There have been 67 TEM $\beta$-lactamase variants identified from clinical isolates up to July 7, 1998 (from the Web site in ref 9). This is the largest group of $\beta$-lactamases, which all trace their descendency to the TEM- 1 enzyme, described first in 1965 (Datta, N.; Kontomichalou, P. Nature 1965, 208, 239).

(13) Miyashita, K.; Massova, I.; Taibi, P.; Mobashery, S. J. Am. Chem Soc. 1995, 117, 11055 .

(14) Maveyraud, L.; Massova, I.; Birck, C.; Miyashita, K.; Samama, J. P.; Mobashery, S. J. Am. Chem. Soc. 1996, 118, 8, 7435. 

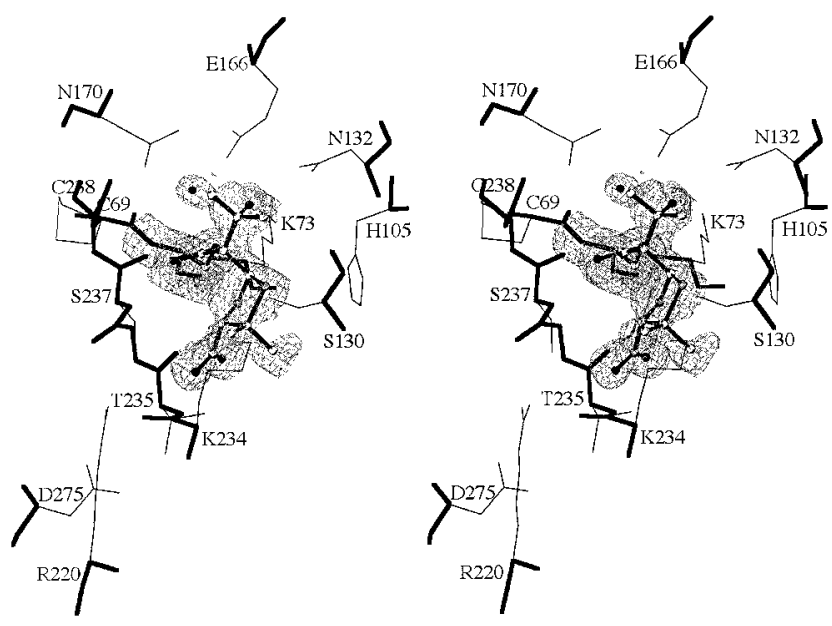

Figure 1. Stereoview of the active site of the crystal structure of the NMC-A $\beta$-lactamase from Enterobacter cloacae NOR-1 modified by 3 . The final $2 F_{\text {obs }}-F_{\text {calc }}$ is contoured at one standard deviation. The main chain is represented as thick lines, the side chains with thin lines. White, gray, and black spheres represent carbon, nitrogen and sulfur, and oxygen atoms, respectively. The hydrolytic water is shown as an isolated black dot at 12 o'clock.

\section{Scheme 1}
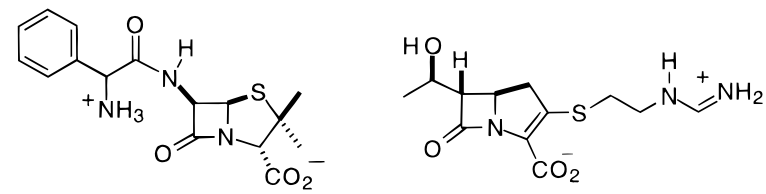

Ampicillin

Imipenem
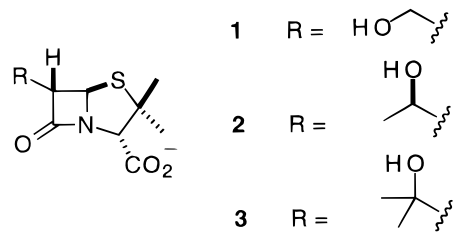

kinetic behavior of this enzyme (Table 1) compared to that of the TEM-1 enzyme. ${ }^{15}$ The crystal of the complex diffracted to $1.9 \AA$ resolution and belongs to the space group $P 2{ }_{1} 22_{1} 2$ with cell parameters $a=77.55 \AA, b=52.46 \AA$, and $c=67.22 \AA .{ }^{15}$ After refinement to $1.9 \AA$, the electron density maps showed all of the features of the inhibitor molecule bound in the active site as an acyl enzyme species (Figure 1) and indicates no conformational change of the protein upon inhibitor binding.

The structure of the NMC-A $\beta$-lactamase is similar to that of other class A enzymes, despite its increased breadth of substrate profile and the rms deviation of the C $\alpha$ carbons of the NMC-A enzyme when compared to the structure of the TEM-1 enzyme is $2 \AA$. The slightly different orientation of the two domains which define the substrate binding site and folding variations in the helical domain of the protein affect the position of helix H5. It contains the active site residue Asn-132, which shifts away from the active site. However, the position of the hydrolytic water, coordinated by residues Glu-166 and Asn-170, is the same as in the TEM- 1 enzyme. The residue 244 is an arginine in most class A enzymes. Arg-244 interacts strongly with the substrate carboxylate in these $\beta$-lactamases. ${ }^{14,16,17}$ On the other hand, an alanine is found at position 244 of the NMC-A enzyme. It is of

(15) The preparations of the crystals of the native NMC-A $\beta$-lactamase and the general structural observations of the enzyme will be published subsequently. The final model of the enzyme-inhibitor complex was composed of all protein atoms, with the exception of the solvent-exposed side chains of Lys-253 and Lys-272, and it included 191 crystallographic water molecules and 3 molecules of 4-morpholineethanesulfonate (MES) buffer molecules. The final $R_{\text {factor }}$ was 0.209 ( $R_{\text {free }}$ was 0.257$)$ for all reflections between 18.6 and $1.9 \AA$. interest that despite this seemingly major difference in the mechanisms of substrate anchoring between the NMC-A enzyme and other $\beta$-lactamases, the general positioning of $\mathbf{3}$ in the active site of the NMC-A enzyme is essentially the same as that seen for 1 with the TEM-1 enzyme. ${ }^{18}$ The ester carbonyl is housed within the oxyanion hole, making hydrogen bonds to the mainchain nitrogens of residues 237 and 70 (2.8 and $2.9 \AA$, respectively).

The major difference in the structures corresponds to that of the respective $6 \alpha$ substituents. The repositioning of helix H5 away from the active site allows the hydroxyl group of the $6 \alpha-$ hydroxypropyl moiety to be at hydrogen-bonding distance to Lys$73 \mathrm{~N} \epsilon(3.0 \AA)$, Asn-132 (a bifurcated hydrogen bond to $\mathrm{O} \delta 1$ and to $\mathrm{N} \delta 2,3.1 \AA$ each), and to the hydrolytic water $(3.0 \AA)$. The steric crowding by the geminal $\mathrm{Me}_{2}$ group protects the ester carbonyl from the approach of the hydrolytic water, accounting for the lack of deacylation seen in the complex (for at least $36 \mathrm{~h}$ ).

The repositioning of Asn-132 enlarges the substrate binding cavity by approximately $1 \AA$, and the side chain in this new position is now poised to make a hydrogen bond with the hydroxyl groups of the $6 \alpha$ substituents of compounds $\mathbf{1 - 3}$, as well as that of imipenem. Modeling from the current X-ray structure indicates that this interaction would leave open the approach of the hydrolytic water at the ester carbonyl of the acyl enzyme intermediate resulting in deacylation of active site bound substrates such as imipenem and $\mathbf{1}$, but the increasing steric encumbrance of the $6 \alpha$ substituents of $\mathbf{2}$ and $\mathbf{3}$ impedes the rate of deacylation for these compounds. The interactions of the hydroxyethyl group of imipenem with the hydrolytic water in the TEM- $1 \beta$-lactamase accounts for its reduced rate of deacylation from that enzyme, ${ }^{19}$ analogous to the mechanism of inhibition of that enzyme by $\mathbf{1}^{13,14}$ This is different in the case of the NMC-A enzyme in light of the discussion on the position of residue 132, permitting the facile deacylation of the acyl enzyme intermediate in this case.

The information on the NMC-A $\beta$-lactamase disclosed here is a hallmark of how a subtle change in the structure of an enzyme could make a significant difference in kinetic properties of that enzyme compared to other related enzymes. In light of the fact that we remain highly dependent on $\beta$-lactam antibiotics in treatment of bacterial infections presently and that it is likely that we will rely on these versatile antibacterials for the foreseeable future,${ }^{20}$ it is incumbent upon us to understand the molecular bases for the resistance to these pharmaceutical agents at the molecular level. This mechanistic and structural information would prove helpful in the design of inhibitors for these deleterious enzymes, an exercise which has been performed successfully for the novel NMC-A $\beta$-lactamase in the present work.

Acknowledgment. Valérie Guillet is acknowledged for her excellent assistance in data collection. The work in France was funded in part by INSERM (CRE contract 930612; J.P.S.) and the Regional Midi-Pyrenees (contract 9200843; J.P.S.). The work in the U.S.A. was supported by grants from the National Institutes of Health and the National Science Foundation (S.M.).

Supporting Information Available: Detailed procedures for syntheses, kinetic determinations, information on data collection, structure determination and refinement, and the image of Figure 1 without the electron density are provided (10 pages, print/PDF). The crystallographic coordinates are deposited in the Brookhaven Protein Data Bank. See any current masthead page for ordering information and Web access instructions.

JA9817996

(16) Zafaralla, G.; Manavathu, E. K.; Lerner, S. A.; Mobashery, S. Biochemistry 1992, 31, 3847.

(17) Strynadka, N. C. J.; Adachi, H.; Jensen, S. E.; Johns, K.; Sielecki, A.; Betzel, C.; Sutoh, K.; James, M. N. G. Nature 1992, 359, 700.

(18) The average $B$ factors are $11.7 \AA^{2}$ for protein atoms $\left(10.8 \AA^{2}\right.$ and $12.7 \AA^{2}$ for main chains and side chains, respectively), $28.8 \AA^{2}$ for Ser-70 acylated by 3,20.1 $\AA^{2}$ for solvent atoms, and $45.1 \AA^{2}$ for MES buffer atoms.

(19) Taibi, P.; Mobashery, S. J. Am. Chem. Soc. 1995, 117, 7600.

(20) Bush, K.; Mobashery, S. In Resolving the Antibiotic Paradox: Progress in Understanding Drug Resistance and Development of New Antibiotics; Rosen, B. P., Mobashery, S., Eds.; Plenum Press: New York, 1998; in press. 